\title{
Hand-foot syndrome in a patient with metastatic lung adenocarcinoma induced by high-dose icotinib: A case report and review of the literature
}

\author{
YULONG ZHENG, WEIJIA FANG and NONG XU \\ Department of Medical Oncology, The First Affiliated Hospital, School of Medicine, \\ Zhejiang University, Hangzhou, P.R. China
}

Received April 18, 2012; Accepted July 27, 2012

DOI: 10.3892/ol.2012.904

\begin{abstract}
Icotinib is a new oral epidermal growth factor tyrosine kinase inhibitor (EGFR-TKI). The most frequent side-effects of icotinib include rash and diarrhea. Hand-foot syndrome (HFS) induced by EGFR-TKI is rare. The present study describes, for the first time, HFS induced by high-dose icotinib in a 65-year old female with metastatic lung adenocarcinoma. The patient developed HFS during the first week of icotinib treatment with characteristic clinical presentation. HFS regressed after icotinib dose-reduction was initiated. HFS may occur with icotinib, especially when administered in high doses.
\end{abstract}

\section{Introduction}

Icotinib hydrochloride is a new, small-molecule, selective epidermal growth factor receptor tyrosine kinase inhibitor (EGFR-TKI) (1-4). A large randomized head-to-head phase III clinical trial (ICOGEN) demonstrated that icotinib has a similar efficacy to gefitinib in previously treated non-small cell lung cancer (NSCLC), with less toxicity (4). The most frequent side effects of icotinib include rash (40.0\%) and diarrhea (18.5\%) (4). Since August 2011, this drug has been available on the Chinese market. The recommended dose is $125 \mathrm{mg}$ TID orally, which is same as that used in the ICOGEN trial.

Hand-foot syndrome (HFS) is a distinctive and relatively common cutaneous toxicity of traditional chemotherapeutic agents, including 5-fluorouracil, liposomal doxorubicin,

Correspondence to: Dr Nong Xu, Department of Medical Oncology, The First Affiliated Hospital, School of Medicine, Zhejiang University, 79 Qingchun Road, Hangzhou, Zhejiang 310003, P.R. China

E-mail: xunong126@126.com

Key words: hand-foot syndrome, epidermal growth factor, kinase inhibitor, adverse drug reactions cytarabine, docetaxel and capecitabine (5), as well as certain oral targeted therapies such as sunitinib and sorafenib (6). HFS induced by EGFR-TKI is uncommon and has been only sporadically reported in the literature (7-9). We report a case of HFS caused by high-dose icotinib, which, to the best of our knowledge, has not been previously described.

\section{Case report}

A 65-year old female (non-smoker) with metastatic lung adenocarcinoma received one cycle of intravenous chemotherapy with pemetrexed $\left(500 \mathrm{mg} / \mathrm{m}^{2}\right.$, on day 1$)$ plus cisplatin (25 $\mathrm{mg} / \mathrm{m}^{2}$, on days 1-3). The patient experienced serious vomiting (NCI-CTC 3.0, grade 3) and refused to continue. The patient then participated in the dose-escalation study of the safety and pharmacokinetics of icotinib after signing an informed consent form. A baseline target and non-target lesions were obtained through contrast-enhanced computed tomography (CT; Fig. 1A).

The patient received icotinib $625 \mathrm{mg}$ TID orally. Five days after the initiation of icotinib, she noticed scattered red, point-like rashes in the palms of the hands and soles of the feet, with a tingling sensation. In the second week, the patient progressively developed serious desquamation in the palms and less in the soles of the feet, accompanied by pain and redness (Fig. 2). Grade 3 HFS was diagnosed. The patient also experienced grade 2 diarrhea and grade 1 rash in the trunk with itching. The dose of icotinib was reduced to $250 \mathrm{mg}$ TID. The patient was prescribed oral vitamin B6 and urea cream for HFS and zyrtec for the trunk rash and pruritus. In the fourth week, the HFS subsided to grade 1 . The trunk rash persisted as grade 1 . The diarrhea resolved spontaneously. A repeated CT demonstrated that the patient obtained a partial remission (PR) of the disease (Fig. 1B). A subsequent CT in the eighth week confirmed the PR. The patient has been undergoing icotinib treatment for 12 months, which continues, and was doing well at the time of writing.

This study was approved by the institutional ethics committee of The First Affiliated Hospital, School of Medicine, Zhejiang University. Informed consent was obtained from the patient and her family. 

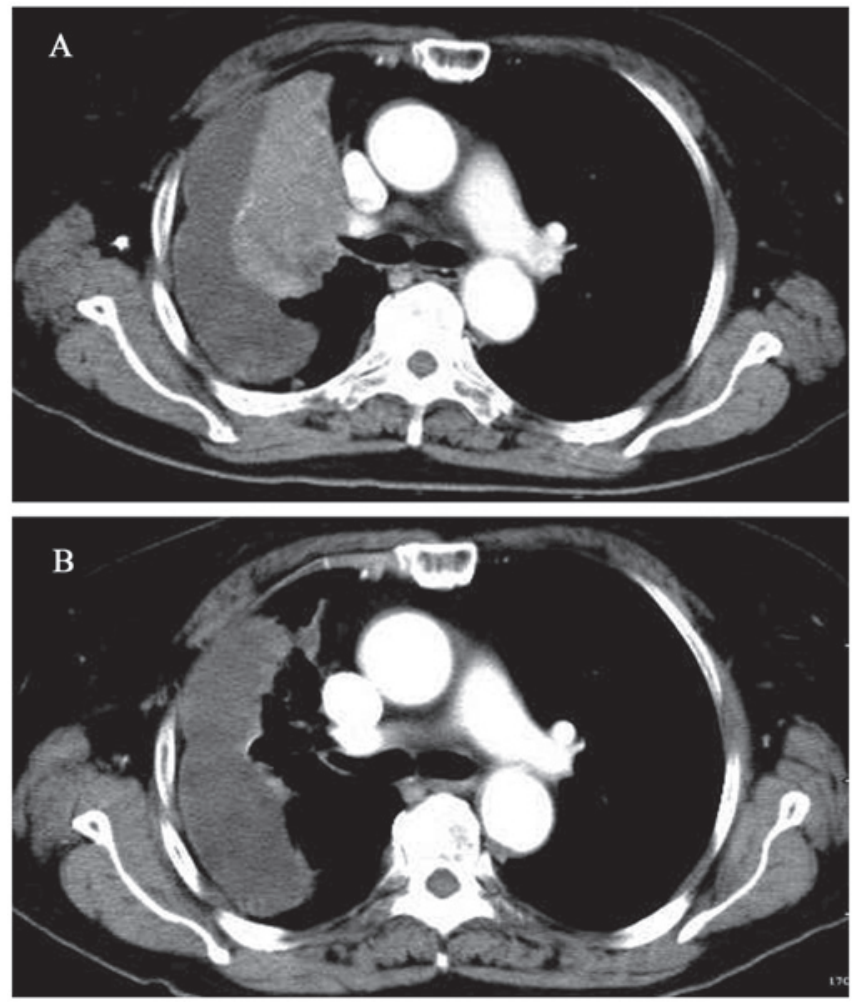

Figure 1. (A) Prior to icotinib treatment, chest CT indicated a huge mass in the right lung and right pleural effusion. (B) Four weeks after icotinib treatment, a repeated CT showed that the huge mass had been mostly absorbed. $\mathrm{CT}$, computed tomography.

\section{Discussion}

HFS is also known as palmar-plantar erythrodysesthesia or acral erythema and is characterized by symmetrical erythema of the palms and soles, together with sores, edema, paresthesia and blisters. In some instances, desquamation and ulceration occur. HFS generally arises after 2-4 weeks of treatment with oral targeted therapies $(6,7,9)$. In the present case, the patient suffered from evident desquamation with pain, interfering with activities of daily living (ADL). The clinical presentation was characteristic; however, the condition was developed during the first week of treatment, which is earlier than normal. We hypothesize that the early exhibition of HFS was induced by high-dose icotinib.

When it occurs following treatment with cytotoxic agents, HFS appears to be dose-dependant and its occurrence seems to be decided by peak drug concentration and total cumulative dose (10). Since 2006, our institute has participated in phase I, II and III clinical trials of icotinib. Learning from this experience, the incidence of HFS in patients with long-term oral icotinib treatment is not elevated. In the present case, the peak drug concentration appears to have been an important factor in the occurrence of HFS. However, the exact correlation remains to be further elucidated.

A wide variety of therapies have been suggested to treat HFS: dose reduction and treatment interruption, COX-2 inhibitors, topical urea-containing creams, pyridoxine supplement, emollient creams, regional cooling and oral corticosteroids. The most definitive treatment is dose modi-
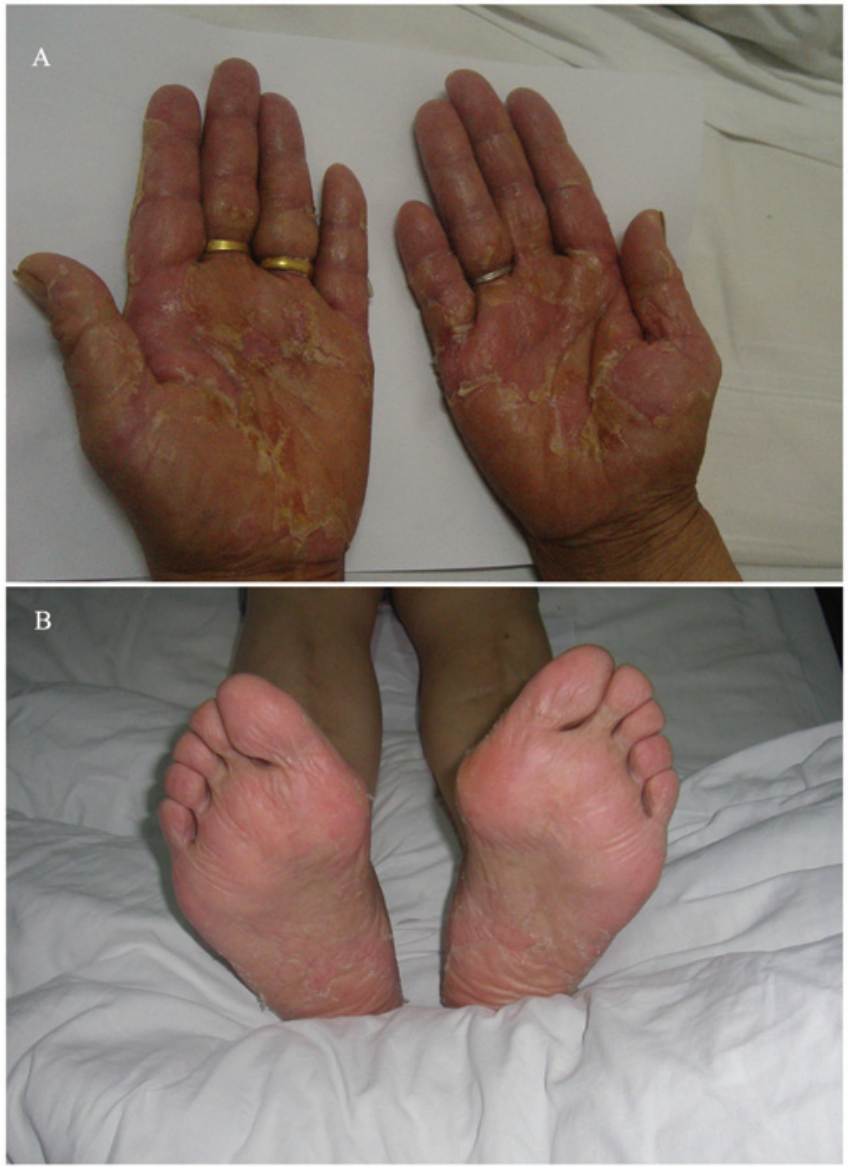

Figure 2. Acral erythema and desquamation on both (A) palms and (B) soles. The symptoms were more severe on the palms.

fication $(5,6)$. We treated the patient with oral vitamin B6 and topical urea cream. A decision of treatment reduction was immediately made when the HFS progressed to grade 3, since the dose-limiting toxicity of icotinib was observed. It is postulated that the rapid regression of HFS is mostly due to prompt dose reduction.

Icotinib and erlotinib share a common chemical backbone structure but differ in their side chains (2); nonetheless, their side-effect profiles are different $(4,11)$. In the two reported patients with HFS induced by erlotinib, dose reduction was ineffective $(7,9)$. In our case, the reduction of icotinib dose contributed most to the regression of HFS symptoms.

Razis et al (8) reported three cases of HFS associated with gefitinib, which the authors considered to be 'recall reactions' to previous exposure to liposomal doxorubicin. In our case, the patient underwent one cycle of chemotherapy with pemetrexed plus cisplatin one month before icotinib treatment. Cisplatin-induced acral erythema has been reported in the literature $(12,13)$. It is unlikely that the HFS was induced by cisplatin in our case. First, the incidence of acral erythema in patients receiving cisplatin is extremely rare, having been reported in only four cases (13). Second, the HFS in the present case began in the first week of icotinib treatment. Furthermore, the symptoms soon regressed when the icotinib dose was reduced. However, excluding the possibility of a 'recall reaction' to previous exposure to cisplatin was difficult in this patient. 
In conclusion, EGFR-TKI-associated HFS is unusual. Icotinib, a EGFR-TKI, may induce the condition, especially when it is administered at a high dose. The syndrome is painful and distressing to patients; in some instances it interferes with ADL and results in dose interruption. Clinicians should consider the syndrome when a patient commences icotinib treatment.

\section{References}

1. Zhao Q, Shentu J, Xu N, et al: Phase I study of icotinib hydrochloride (BPI-2009H), an oral EGFR tyrosine kinase inhibitor, in patients with advanced NSCLC and other solid tumors. Lung Cancer 73: 195-202, 2011.

2. Guan Z, Chen X, Wang Y and Zhong D: Metabolite identification of a new antitumor agent icotinib in rats using liquid chromatography/tandem mass spectrometry. Rapid Commun Mass Spectrom 22: 2176-2184, 2008.

3. Liu D, Jiang J, Hu P, Tan F and Wang Y: Quantitative determination of icotinib in human plasma and urine using liquid chromatography coupled to tandem mass spectrometry. J Chromatogr B Analyt Technol Biomed Life Sci 877: 3781-3786, 2009.

4. Sun Y, Shi Y, Zhang L, Liu X, Zhou C, Zhang L, Wang D, Li Q, Zhang S, Qin S, et al: A randomized, double-blind phase III study of icotinib versus gefitinib in patients with advanced non-small cell lung cancer (NSCLC) previously treated with chemotherapy (ICOGEN). J Clin Oncol 29 (Suppl): abstr 7522, 2011.

5. Webster-Gandy JD, How C and Harrold K: Palmar-plantar erythrodysesthesia (PPE): a literature review with commentary on experience in a cancer centre. Eur J Oncol Nurs 11: 238-246, 2007.
6. Lacouture ME, Wu S, Robert C, et al: Evolving strategies for the management of hand-foot skin reaction associated with the multitargeted kinase inhibitors sorafenib and sunitinib. Oncologist 13: 1001-1011, 2008

7. Benomar S, Boutayeb S, Afifi Y, et al: Hand-foot syndrome and seborrheic dermatitis-like eruption induced by erlotinib. Dermatol Online J 15: 2, 2009.

8. Razis E, Karina M, Karanastassi S and Fountzilas G: Three case reports of hand-foot syndrome with gefitinib. Cancer Invest 24: 514-516, 2006.

9. Rouxel AM, Roguedas AM, Descourt R and Misery L: Hand-foot syndrome: A new side effect of erlotinib. Ann Dermatol Venereol 135: 762-764, 2008 (In French).

10. Nagore E, Insa A and Sanmartin O: Antineoplastic therapyinduced palmar plantar erythrodysesthesia ('hand-foot') syndrome. Incidence, recognition and management. Am J Clin Dermatol 1: 225-234, 2000.

11. Shepherd FA, Rodrigues Pereira J, Ciuleanu T, et al; National Cancer Institute of Canada Clinical Trials Group: Erlotinib in previously treated non-small-cell lung cancer. N Engl J Med 353: $123-132,2005$.

12. Vakalis D, Ioannides D, Lazaridou E, Mattheou-Vakali G and Teknetzis A: Acral erythema induced by chemotherapy with cisplatin. Br J Dermatol 139: 750-751, 1998.

13. Fukamachi S, Nakamura $M$ and Tokura Y: Cisplatin-induced acral erythema. Eur J Dermatol 19: 171-172, 2009. 